\title{
Outer Segment of the Photoreceptor Cell
}

National Cancer Institute

\section{Source}

National Cancer Institute. Outer Segment of the Photoreceptor Cell. NCI Thesaurus.

Code C33239.

The distal portion of the photoreceptor cells made up of densely packed disks containing opsin proteins that absorb light. 